\title{
THE EFFECT OF SELF-ASSEMBLING NANOSTRUCTURES BY CIRCULARLY POLARIZED LIGHT: REVIEW PAPER
}

\author{
Rashid Nizam \\ Department of Physics, IFTM University, \\ Moradabad, India
}

\author{
Adeeba Zafar \\ Department of Physics, IFTM University, \\ Moradabad, India
}

\begin{abstract}
Spin angular momenta of circular polarized photons influenced chemical reactions. It has revealed that the circular polarized light (CPL) strongly influences self-assembly of racemic CdTeNps. When light is enlightened to sample of racemic CdTe NPs, NPs separated in to right-and left- handed twisted nanoribbons with right- and left- handed circular polarized light (CPL) respectively which induces due to light. This is because of spin angular momenta of circular polarized photons influenced chemical reactions. It is seen that enlightening to such chemical reactions surplus both type of enantiomers which is 10 times higher than CPLinduced reactions. Further illumination of linearly polarize light plus assembly in dark led to non-twisted nanoribbons. The mechanism of "templation"of NPs assemblies by CPL is correlated with selective photo-activation of chiral NPs moreover clusters followed by their photo-oxidation. Chiral anisotropy interfaces translate in to chirality of the given assembled ribbons. The ability of NPs to preserve polarization information of incident photon releases new pathways for the synthesis of chiral photonic materials. It also allows for improvement understanding of the origins biomolecular homo-chirality.
\end{abstract}

Keywords: Chirality; self - assembly; circularly polarized light; nanoribbon; chiroptical properties

\section{INTRODUCTION}

Nanoscale material strongly rotates polarisation of linearly polarised light (LinP) and circular polarised light. The opposite effect that is the transfer of spin angular momenta of circularly polarised photons to matter and its subsequent nanoscale or atomic restructuring, retaining the "memory" of circular polarisation are much less known ${ }^{1-8}$. For such effect, the formation of spiral nano needles with controlled helicity during laser ablation of bulk metallic tantalum with CPL, the effect of this mechanism is not known properly [9]. In micro scale collides ${ }^{10}$ ensembles of cold atoms [11] spin angular momenta transfer into the focused into the laser beam having high intensity which is also observed in boss Einstein condensates [12]. High intensity of CPL of light up these scatters which occur circular or spiral motion during this. Media vanishes rotational motion very fast which makes it difficult translation of physical motion into the permanent structure changes of these atoms and particular system despite of achiral symmetry, there structure resist the transmission of rotational energy into chemical bond.

For an extensive molar misses and sizes of particles, the transfer of spin angular momenta was observed between the range from cold atom ensembles $\mathrm{M} \sim 10^{2}$ to micro scale collides $\mathrm{M} \sim 5 \times 10^{12}$. Mass and size of nanoparticles which are closer to Boss-Einstein condensates $\left(\mathrm{m} \sim 10^{7}\right)$ [12]. and atom clusters, micro particles of some CPL effect showed be observed it.

Expectation that of CPL could possibly effected self-assembly mechanism of nanoparticles are based on chirality of nanoparticles which is measured at atomic and nanoscale..$^{13}{ }^{14}$ In NPs assemblies build-up of circular polarization..$^{15}{ }^{16}$ To little changes in inter particles interactions process of responsiveness of self-assembly. ${ }^{17}$ In the light of above hypothesis, under fixed parameters assembly of NPs like CdTe soluble in water effected by CPL.

\section{PROCEDURE}

In the light of above hypothesis, under fixed parameters assembly of NPs soluble in water effected by CPL.With range $543 \mathrm{~nm}$ wavelength either RCP or LCP elucidate CdTe. NPs dispersion getting stability by the achiral capping agent thioglycolic acid (TGA). Note that there were no circular dichroism peaks shown in visible range and LCP, RCP with 543 wavelength having 


\section{International Journal of Engineering Applied Sciences and Technology, 2019 Vol. 4, Issue 5, ISSN No. 2455-2143, Pages 175-179 \\ Published Online September 2019 in IJEAST (http://www.ijeast.com)}

tantamount absorbance. From the illumination the previous study ${ }^{18}$ expectation of build of twisted nanoribbons was unclear. Transformation of nanoparticle with CPL observed after $1 \mathrm{~h}, 12 \mathrm{~h}, 28 \mathrm{~h}$, $35 \mathrm{~h}$ and $50 \mathrm{~h}$ (fig $1 \mathrm{~A} \& 1 \mathrm{~B}$ ). Products of 50-200 $\mathrm{nm}$ (1h) rods were included in temporary age which developed 1-2 $\mu \mathrm{m}$ twisted nanoribbons (12h) and further transformed into $2-3 \mu \mathrm{m}$ nanoribbons (28h). With total length, pitch length and diameters of $3 \pm 0.5 \mu \mathrm{m}, 800 \pm 20 \mathrm{~nm}$ and $50 \pm 5 \mathrm{~nm}$ resp. twisted nanoribbons were one of the important products of the photo induced reaction after 50h (fig 1C). When passed CPL in nanoribbons for 50h, they did not lose their obtained twisted shape but started to be thinner.

They have two properties, a different photo chemical effect on NP dispersion an outcome geometry of twisted nanoribbon displayed by CPL. With LCP maximum products were left handed twisted nanoribbons. Similarly, with RCP right handed nanoribbons as estimated by SEM or similar dimensions strong (fig 1B). Circular dichroism spectroscopy also confirmed chirality in nanoribbons. CD spectra of pure nanoribbons were obtained in aqueous dispersion and at 490, 590 and 700 showed different chiro optical bonds Nanoribbons dispersion with RCP and RCP revealed negative and positive $\mathrm{CD}$ signals resp. TEM tomography visualized 3D chiral shape of nanoribbons (fig 2A \& B). Including illumination, right and left handed nanoribbons had equally distribution of UnP and with LinP as well as in dark yielded straight nanowires. Above experiment did not revealed CD activity.

Another experiment, with a different light source, dispersion of CdTe at $607 \mathrm{~nm}$ for 50h drops 20\% efficiency of induction by LCP is decrease as the difference between $\mathrm{LH}$ and $\mathrm{RH}$ nanoribbons compared to $543 \mathrm{~nm}$.

Possible images of small molecular weight compound arising the effect of chirality. To avoid this effect taken for single nanoribbon (fig $2 \mathrm{E} \& 2 \mathrm{~F})$. For the $600-800 \mathrm{~nm}$ spectral window dissimilarity between $\mathrm{CD}$ measurement and $\mathrm{CD}$ spectroscopy of chiral structure ensembles in dispersion.

The single LH and RH nanoribbons showed likely positive and negative mirror-image CD signals. A band located at $600-700 \mathrm{~nm}$ is spectrally similar to the band observed to the dispersion (fig 1F). Using microscopy in dark measured the CD spectra of single twisted nanoribbons are controlled by scattering. With specific handedness, scattering of RCP and LCP photons on twisted nanoribbons possess the 'red' CD bands (fig 1F). Against potential artifacts, from single nano ribbon at various rotational angles in respect to the long access measured CD spectra. Incident angle has no effect on shape of CD bands. As another control of experiment, CD signals did not show by straight nano wire.

Polarization rotation can be compared to the chirooptical properties of the twisted nano ribbon. Anisotropy factor of $\mathrm{g}=0.02-0.04$ were obtained from numerical FEM solution of Maxwell equation for CdTe twisted ribbons needles (fig 2C \&2D) which is comparable to the values obtained for super $\mathrm{AG}$ enhanced $\mathrm{Au}$ and $\mathrm{Au}$ nanorods /fiber composites. g 0.025 . [2,3].

Different parameters differences in shape nanoribbon due to polarization of photons. Perceive, Firstly, the mechanism of light induced self-assembly of CdTe NPs. Parallel light stimulation is the process of formation of twisted nanoribbon with the spontaneous self-assembly of NPs revealed by the optical \& relative properties of nanoparticles in supernatant of nanoribbon dispersions made with \& without light $[18,19]$. FTIR peaks $v(\mathrm{O}-\mathrm{H}), \quad \delta(\mathrm{O}-\mathrm{H}), \quad v_{\mathrm{s}}(\mathrm{COOH})$, characteristic of TGA ligand located at $3500 \mathrm{~cm}^{-1}$ and $1421 \mathrm{~cm}^{-1}$ resp. were decreased in nanoribbons compared to the original CdTe (fig.3A). After the separation of twisted nanoribbons and NPs obtained a strong peak $v_{a s}$ (COO-) of carboxyl moieties at $1722 \mathrm{~cm}^{-1}$. Decomposition of TGA due to the illumination time increased as well as the characteristic of UV-Vis absorption peak of TGA at $276 \mathrm{~nm}$ decreased. Replacement of Te in NPs with $\mathrm{S} 2 \mathrm{p}$ signal from CdS in X-ray photoelectron spectra (XPS) of nanoribbons because weakness for Te peaks from CdTe. TEM of the twisted region, energy dispersive spectroscopy (EDS)and atomic mapping images confirmed the transformation of CdTe into CdS (Fig. 3B). these results related to the formation of a thin $\mathrm{CdS}$ shell around CdTe NPs[20].

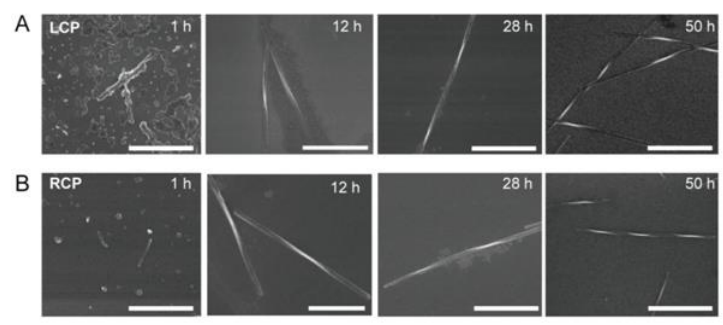




\section{International Journal of Engineering Applied Sciences and Technology, 2019 Vol. 4, Issue 5, ISSN No. 2455-2143, Pages 175-179 \\ Published Online September 2019 in IJEAST (http://www.ijeast.com)}
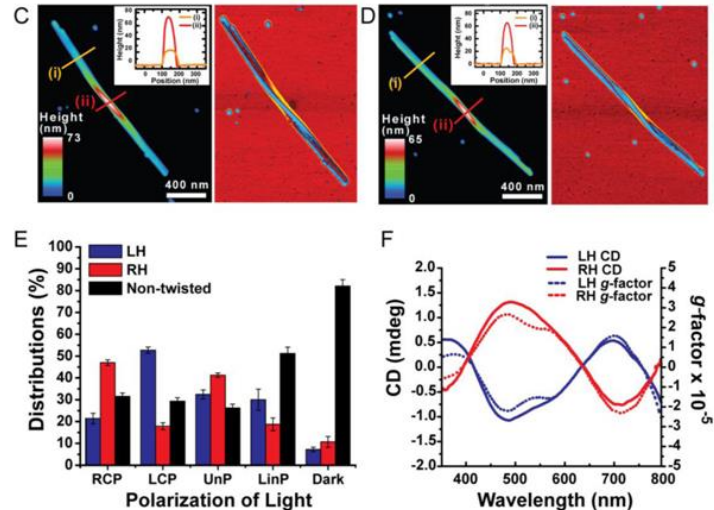

Figure 1. Self-assembly of CdTe NPs into twisted nanoribbons induced by illumination with CPL (Fig Aand B), images of the ribbons assembled with LCP (A) and RCP (B) by SEM as a function of time exposure for $1 \mathrm{~h}, 12 \mathrm{~h}, 28 \mathrm{~h}$ and $50 \mathrm{~h}$. All scale bars are $1 \mu \mathrm{m}$. (FigC and D), tapping mode atomic force microscopy (AFM) phase (right) andtopography (left) images of LH nanoribbon (C) and RH nanoribbon (D). (FigE), Distributions of $\mathrm{RH}, \mathrm{LH}$, and non-twisted nanoribbons obtained after $50 \mathrm{~h}$ illumination with RCP, LCP, LinP, UnP light, and in the dark. (Fig F), At CPL illumination,ensemble CD spectra (solid line) and $g$ factor (dotted line) of dispersions of right-handed $(\mathrm{RH})$ nanoribbons and left-handed (LH) nanoribbons obtained after $50 \mathrm{~h}$. Linear dichroism shows that could be associated with adsorption on the walls of the cuvette and other spontaneous alignment of linear nanostructures have negligible contribution to the chiroptical properties as point out by the identity of the CD spectra obtained with and without action of the dispersion

Based on the above result, CdTe clusters and NPs can be chiral. In racemic mixtures chirality of individual Au NPs capped with achiral ligands as TGA formerly reveal liquid chiral chromatography ${ }^{21}$. Similarly NPs stabilized by TGA, high angle annular dark field (HAADF) scanning transmission electron microscopy (STEM) (Fig 3G Fig 3H) confirmed shape of CdTe-TGA NPs also revealed by high resolution TEM images (Fig. 3I). At the fixed parameter the dispersion of CdTe NPs stabilised by TGA with enantioselective selective adsorber Bromine serum albumin (BSA). After 5h, using centrifugal membrane $(50 \mathrm{kDa})$ they separated $\mathrm{BSA}$ and unbounded NPs then measured CD spectra of dispersions. The original CdTe NPs has no CD signals but BSA has negative CD peak at $215 \mathrm{~nm}$ and separated NPs revealed positive CD bands at $400-550 \mathrm{~nm}$. Based on the above study a racemic mixture of NPs is the starting NP dispersion and it can be separated by enantioselectively.
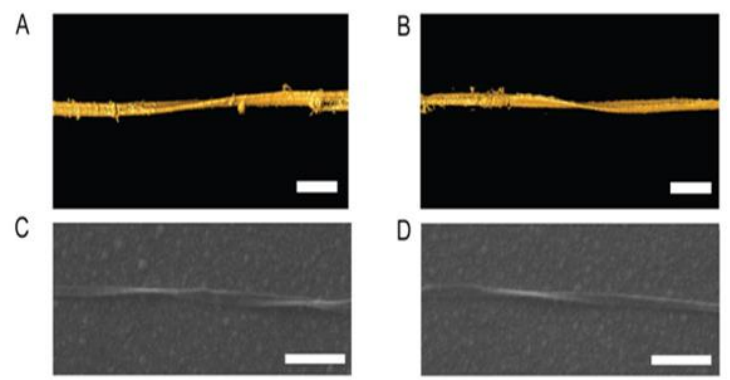

Figure 2. Chirality of single nanoribbons (Fig Aand B), Surface exposition of 3D TEM tomographic reconstruction of LH nanoribbon (A) and RH nanoribbon (B). Scale bars are $100 \mathrm{~nm}$. (Fig C and D), SEM images of single $\mathrm{LH}(\mathrm{C})$ and RH nanoribbons (D). Scale bars are $500 \mathrm{~nm}$. (Fig E and F), CD spectra and calculated $g$ factor spectra for single LH (E) and RH (F) nanoribbons in $\mathrm{C}$ and $\mathrm{D}$, respectively. (FigG and $\mathrm{H}$ ), Computational models of the LH $(\mathrm{G})$ and $\mathrm{RH}(\mathrm{H})$ nanoribbons used in the FEM calculations of chiroptical propertiesdepend on numerical solutions of Maxwell equations.

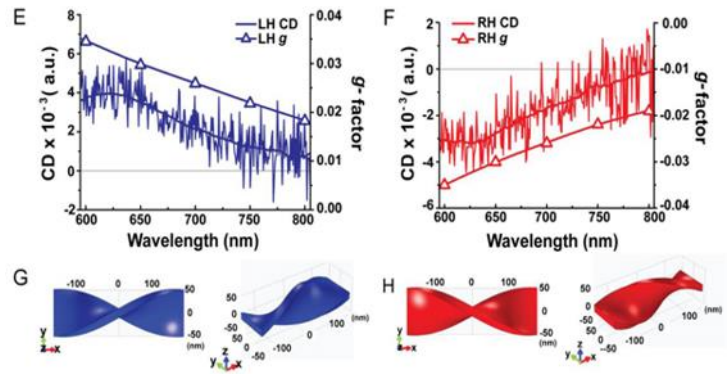

Light absorption revealed that reflection optical activity displayed by enantiomers of truncated NPs in mirror imaged simulated $\mathrm{CD}$ and g-factor spectra (fig. 3D). In such clusters LCP and RCP absorption differences (fig. 3F) are greater than NPs (3D) .
A
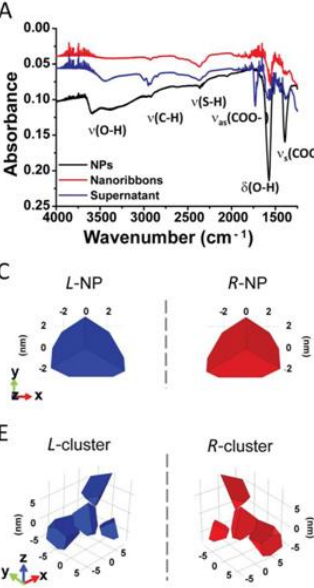

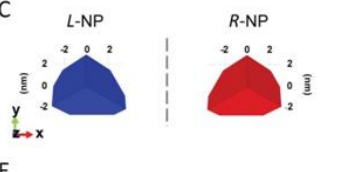

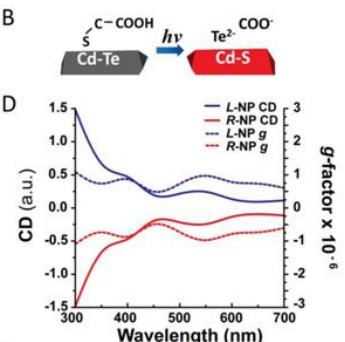

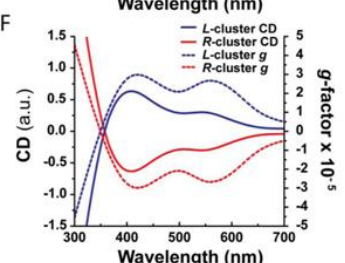




\section{International Journal of Engineering Applied Sciences and Technology, 2019 Vol. 4, Issue 5, ISSN No. 2455-2143, Pages 175-179 \\ Published Online September 2019 in IJEAST (http://www.ijeast.com)}
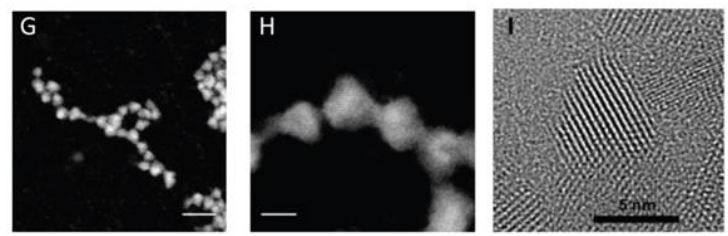

Figure 3. Mechanism of enantioselective assembly of NPs (Fig A), After $50 \mathrm{~h}$ of illumination time obtainedFTIR spectra of original CdTe NPs, supernatantand purified nanoribbons. (FigB), Schematic demonstration of CdTe phase transition to CdS. (Fig C and E), Models of chiralNPs (C) and chiral NP clusters (E) used in determining of chiroptical properties. (FigDand F), Simulated spectra and $g$-factors for (D) $L / R$-NPs and (F) $L / R$-clusters of NPs. Nomenclature forNPs and their clusters is dependent on the negative $(R)$ and positive $(L)$ optical activity. (Fig Gand H), HAADF STEM images of TGA-stabilized truncated tetrahedral CdTe NPs. (FigI), High resolution TEM image of TGAstabilized truncated tetrahedral CdTe NPs . Scale bars are $15 \mathrm{~nm}(\mathrm{G})$ and $5 \mathrm{~nm}(\mathrm{H})$.

The chirality transfer mechanism from CPL to NP assemblies can described as- when LCP passed in a racemic mixture of CdTe NPs at 543nm then $\mathrm{LH}$ NPs/clusters more effectively absorb the light compared to RH NPs and the same is true for RH NPs and clusters. The light-activated CdTe NPs go through photo-oxidation TGA stabilizes transform into "bare" CdS NPs. The ligand free CdS NPs revealed stronger property to self-assemblies than non-light activated, ligand-protected CdTe NPs. Because for the anisotropy of NPinteractions the self-assembly of NPs. To clarify the origin of ribbon helicity atomic molecular dynamic (MD) were performed. Individual NPs has $3.6 \mathrm{~nm}$ tetrahedrons shape with cubic CdS crystals lattice and lattice constant of $a=0.582$ (Fig. 4A). By the removal of $2,3 \& 4$ atom layers, the tetrahedrons were asymmetrically truncated from three of the NPs vertices to a right or left chirality.

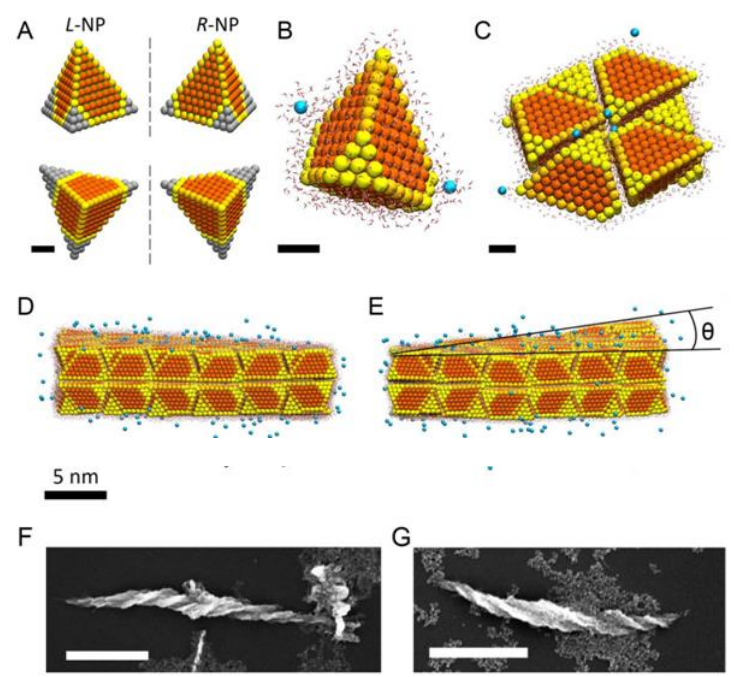

Figure 4. Molecular dynamic and experimental studies of self-assembly of chiral NPs Scale bars in A, B, C are $1 \mathrm{~nm}$. (Fig A), Atomistic models of NPs with RH and LH truncations used in MD simulations. (FigB), showsa single NP in aqueous environment with counter ions used in MD simulations. (FigC), A fragment of the simulated self-assembled ribbon from (top view) revealed packing of NPs. (FigDand E), The side views of simulated NP ribbon with LH (D) and RH (E) truncated NPs. The pitch of the nanoribbons based on Dihedral angle $\theta$. (FigFand G), SEM images of experimental assemblies spontaneously formed in murk from chiral CdTe NPs stabilized by L-cysteine (F) and D-cysteine (G).

At equilibrium condition NPs assemblies at $\mathrm{T}=300$ $\mathrm{K}$ for $\sim 5$ to $10 \mathrm{~ns}$ in an isothermal - isobaric ensemble, the planar nanoribbon used to take different twist. Importantly for NPs twist was opposite with opposite handedness. When nanoribbon made from CdS NPs the average twist angle -2.4 and $+4.6^{\circ}$ for $\mathrm{LH}$ and RH NPs(Fig. 4D and Fig 4e) were observed respectively and the pitch length of $\sim 600-800 \mathrm{~nm}$.

In the illumination of above experiment, there is fine differences in interparticle interactions between a priori chiral NPs which make nanostructure with different chirality and they can be found for NPs with TGA made from D- and Lcysteine (Fig. 4F, and Fig. 4G).

\section{CONCLUSION}

The Imprint of CPL can be observed on nanoscale structures by nanoparticles of altering chirality which are participating in the process of selfassembly because sensitivity of light is common for nanoparticles. This study opens new synthetic pathways for chiral nanostructures utilizing light as primary parameter controlling the asymmetry in the enantiomeric mixture of the products.

\section{REFERENCES}

[1] MX R., Plum E, Xu J. J., Zheludev N.I. (2012) Giant nonlinear optical activity in a plasmonic metamaterial. Nat Commun.; (3:833.)

[2] Kuzyk A, et al. (2012) DNA-based selfassembly of chiral plasmonic nanostructures with tailored optical response. Nature. (483: pp 311-314.)

[3] Guerrero-Martinez A, et al. (2011) Intense Optical Activity from Three-Dimensional Chiral Ordering of Plasmonic Nanoantennas. Angew Chem Int Edit. (50: pp 5499-5503.) 


\section{International Journal of Engineering Applied Sciences and Technology, 2019 \\ Vol. 4, Issue 5, ISSN No. 2455-2143, Pages 175-179 \\ Published Online September 2019 in IJEAST (http://www.ijeast.com)}

[4] Liu S, et al. (2012) Synthesis of chiral $\mathrm{TiO} 2$ nanofibre with electron transitionbased optical activity. Nat Commun.;

[5] Hentschel M, et al. (2012) Optical Properties of Chiral Three-Dimensional Plasmonic Oligomers at the Onset of Charge-Transfer Plasmons. ACS Nano (6: pp 10355-10365.)

[6] Mark AG, Gibbs JG, Lee TC, Fischer P. (2013) Hybrid nanocolloids with programmed three-dimensional shape and material composition. Nat Mater. (12: pp 802-807.)

[7] Hendry E, et al. (2010) Ultrasensitive detection and characterization of biomolecules using superchiral fields. Nat Nanotechnol. ( 5: pp 783-787.)

[8] Gansel J K, et al. (2009) Gold Helix Photonic Metamaterial as Broadband Circular Polarizer. Science. ( 325: pp 1513-1515.)

[9] Toyoda K., Miyamoto K., Aoki N., Morita R., Omatsu T. (2012) Using Optical Vortex To Control the Chirality of Twisted Metal Nanostructures. Nano Lett. (12: pp 3645-3649.)

[10] Padgett M, Bowman R. (2011) Tweezers with a twist. Nat Photonics. (5: pp 343348.)

[11] Tabosa JWR, Petrov DV. (1999) Optical pumping of orbital angular momentum of light in cold cesium atoms. Phys Rev Lett. (83: pp 4967-4970.)

[12] Brachmann JFS, Bakr WS, Gillen J, Peng A, Greiner M. (2011) Inducing vortices in a Bose-Einstein condensate using holographically produced light beams. Opt Express.; (19: pp 12984-12991). [PubMed: 21747450]
[13] Gautier C, Burgi T. (2006) Chiral Nisobutyryl-cysteine protected gold nanoparticles: Preparation, size selection, and optical activity in the UV-vis and infrared. J Am Chem Soc. (128: pp 11079-11087.)

[14] Chen CC, et al. (2013) Three-dimensional imaging of dislocations in a nanoparticle at atomic resolution. Nature. (496: pp 74.)

[15] Govorov A. O., Fan Z. Y., Hernandez P., Slocik J. M. , Naik R. R. (2010) Theory of Circular Dichroism of Nanomaterials Comprising Chiral Molecules and Nanocrystals: Plasmon Enhancement, Dipole Interactions, and Dielectric Effects. Nano Lett. (10: pp 1374-1382.)

[16] Moshe A. B., Szwarcman D., (2011) Markovich G. Size Dependence of Chiroptical Activity in Colloidal Quantum Dots. ACS Nano (5: pp 9034-9043.)

[17] Talapin DV, et al. (2009) Quasicrystalline order in self-assembled binary nanoparticle superlattices. Nature. (461: pp 964-967.)

[18] Srivastava S, et al. (2010) LightControlled Self-Assembly of Semiconductor Nanoparticles into Twisted Ribbons. Science. (327: pp 1355-1359.)

[19]Tang Z. Y., Kotov N. A., Giersig M. (2002) Spontaneous organization of single CdTe nanoparticles into luminescent nanowires. Science. (297: pp 237-240.)

[20] Gaponik N, et al. (2002) Thiol-capping of CdTe nanocrystals: An alternative to organometallic synthetic routes. J Phys Chem B. (106: pp 7177-7185.)

[21] Dolamic I, Knoppe S, Dass A, Burgi T. (2012) First enantioseparation and circular dichroism spectra of Au38 clusters protected by achiral ligands. Nat Commun. ( 3: pp 798.) 